\title{
DisABILITy PROFILE OF GRANT RECIPIENTS IN A Semi-Rural Area IN South AFricA
}

\begin{abstract}
A descriptive study was undertaken to establish, amongst others, the demographic details and the disability profile of a group of persons with disabilities who collected their disability grant at the Kleinmond Post Office in June 1999. Data were collected by means of a questionnaire during face-to-face interviews with the grantees and/or their proxies. A questionnaire developed for this purpose included a disability catalogue based on the International Classification of Impairments, Disabilities and Handicaps (ICIDH). The repeatability of the questionnaire was $96 \%$.

The mean age of the mainly male grantees was 42 years (range: 18 -64).

Nearly a third of the subjects had been disabled since birth, reinforcing the importance of disability prevention programmes as outlined in several policy documents. Most persons with disabilities had problems with activities in the situational disability category (82\% or 24/29), while only a few reported personal care disabilities (34\% or I0/29). The majority of the grantees $(86 \%$ or $25 / 29)$ reported disabilities in more than one category. This may be an indication that only persons with multiple disabilities qualify for permanent disability grants in South Africa.
\end{abstract}

\section{KEY WORDS: DISABILITY, GRANTS, SEMI-RURAL.}

\section{Acknowledgements: \\ Statistical advice: Mrs F Little, Department of Statistical Sciences, University of Cape Town; Financial assistance: Medical Faculty, University of Stellenbosch.}

\section{INTRODUCTION}

Why is it of interest to physiotherapists to know which disabilities are prevalent in persons who receive a permanent disability grant? Firstly, clients often consult physiotherapists regarding their eligibility for disability grants. Secondly, in view of primary health care delivery in South Africa, it is the role of the physiotherapist to deliver communitybased rehabilitation services to persons with disabilities. Being able to describe aspects of a select group of persons already receiving permanent disability grants can assist the physiotherapist when counselling his/her clients and also in planning appropriate physiotherapy and other services within a primary health care team.

There are many definitions of disability, mainly based on conceptual differences, the most common being that used by the World Health Organisation (WHO, 1980). The WHO is currently in the process of finalising a revised version of the ICIDH, the International Classification of Functioning, Disability and Health, ICIDH-2 (WHO, 1999, www.who.intllicidh). This reviewed document is likely to radically change disability research in the future and the altered terminology should make classification less prejudicial.

The original WHO's International Classification of Impairments, Disabilities and Handicaps (ICIDH) differentiates between three domains of disablement and rests on the premise that there is a linear relationship between impairments, disabilities and handicaps. This is however not true, as a person who presents with an impairment does not necessarily have a disability nor a handicap. It is therefore of concern to note that the application form (VRT-3), which has to be completed by the examining medical officer when a person applies for a disability grant, is based mainly on impairments rather than disabilities, reflecting the original ICIDH premise. Hence, although the term "disability grant" suggests otherwise, an individual's impairments, rather than disabilities are of primary concern to the Department of Social Security, which administers these and other social grants.
One aim of this study was therefore to establish the disability profile of a defined group of recipients of a permanent disability grant, including demographic information that would be helpful in interpreting their disability profile. Other aspects investigated (such as utilisation of a caregiver, health and social needs) will be reported elsewhere.

\section{METHODOLOGY}

The protocol was submitted to and approved by the Research Committee C of the Medical Faculty of the University of Stellenbosch.

Sampling procedures were unnecessary as the study population consisted of all persons with disabilities $(n=32)$ who

CORRESPONDENCE TO:

A. Frieg

Department of Physiotherapy, Faculty of Health Sciences, University of Stellenbosch P.O. Box 19063, Tygerberg 7505 Tel: (021) 938-9304 (w) (021) 552-2445 (h) 
collected their permanent disability grant at the Kleinmond Post Office in June 1999. Previous disability studies have focussed primarily on disability prevalence in rural and urban areas (www.statssa.gov.za; Schneider et al, 1999; Coetzee, 1997; Concha and Lorenzo, 1993; Cornieljie et al, 1993; Mc Laren et al, 1987; Disler et al, 1986). There is however, a paucity of epidemiological information about groups of persons with disabilities in semi-rural areas in the Western Cape. This fact, together with logistical considerations, i.e. time and financial constraints, prompted the researcher to choose the geographical area of Kleinmond, a typical semi-rural town in the Western Cape.

A questionnaire was developed which aimed at establishing, amongst others, demographic characteristics, a disability profile, caregiver reliance and needs of the subjects. The disability catalogue of the questionnaire was based on the International Classification of Impairments, Disabilities and Handicaps (ICIDH) (WHO, 1980) as at the time of planning the study and the submission of the protocol for approval, the newer version, ICIDH-2 (WHO 1999, www.who.intll icidh), was not available to the researcher. Furthermore, application and approval of disability grants in South Africa continues to be based on the original source document.

In the original source document an impairment suggests a deviation within the biomedical status of an individual, while a disability refers to the activities an individual is unable to perform. A handicap on the other hand, relates to a decrease in the fulfilment of the roles a person has on a societal level (WHO, 1980). All three domains are divided into categories and the ICIDH allows for coding of the various aspects. Subsequent to this study the ICIDH-2 was perused by the researcher (ICIDH-2 workshop, 2000). Once the ICIDH-2 is formally ratified by the WHO and there is clarity on the disability movements' opinion regarding the new classification system, it is recommended to use the activity catalogue for similar studies, i.e. establish an activity profile.

The disability catalogue of the questionnaire in the present study consisted of seven categories, i.e. behavioural-, communication-, personal care-, locomotor-, body disposition-, dexterity- and situational disabilities. Each category included a number of activities and subjects were asked to indicate whether or not they experienced problems with any of the activities. Nominal variables, i.e. dichotomous yes/no responses were utilised. In the case of activities which were not applicable to a particular respondent, e.g. "able to care for your children", a third response option ("not applicable") was given. Activities were also recorded as non-problematic when a person could perform the activity with an assistive device in his or her possession, e.g. reading glasses.

A pilot study was conducted in the Caledon/Botriver area (a semi-rural area similar to that of the study area) and the questionnaire was translated into Afrikaans by senior physiotherapists proficient in both English and Afrikaans and the disability field.

All subjects were visited in their homes by the researcher in June/July 1999. The aims of the study were explained to them and/or their proxies, confidentiality was guaranteed and they were interviewed in their home language, once written consent had been given. Only self- or proxy reported disability was recorded and no attempt was made to quantify the extent or severity of the disabilities or to objectively measure abilities.

Four subjects were randomly selected and interviewed on a second occasion in order to assess the repeatability of the questionnaire.

Data were analysed with the statistical software package Statistica.

\section{RESULTS \& DISCUSSION}

A response rate of $90 \%(29 / 32)$ was considered adequate for a study of this nature, as the study population was sufficiently represented to ensure that the results were valid for this group of disability grant recipients.

Reliability of the questionnaire was tested by randomly selecting four grantees and interviewing them a second time. Of all responses of those four subjects, $96 \%$ were the same at both interviews, attesting to a strong likelihood that data were collected in a reliable manner

Grantees contributed to $72 \%(21 / 29)$ of all interviews. In $48 \%$ of cases (14/29) the grantees only were interviewed, while both the grantee and the proxy were interviewed in $24 \%$ (7/29) of cases.

The relationship of the proxy to the grantee in this study population was mainly parent to child. Although proxy reporting may lead to bias (Sackett, 1979), it can be argued that in the present study however, the proxies had intimate knowledge of the persons with disabilities and their needs, and could contribute meaningfully to the interviews. While Schofield et al (1997) agree that carer reports are valid, Knapp and Hewison (1999) reported the opposite.

The median age of the grantees was 40 years (range: 18-64 years). This well-defined range reflects present legislation which determines that disability grants are only paid to adults from 18 years of age, up to 60 and 65 years for women and men respectively. Thereafter, the grants are converted into old age pensions.

The gender distribution of the subjects was $55 \%(16 / 29)$ male and $45 \%$ (13/29) female. Although most grantees (59\% or $17 / 29$ ) were single, the majority (93\% or 27/29) did not stay alone. This may be explained by the fact that the majority of disability grant recipients in this study population (66\% or 19/29) relied to some extent on a caregiver.

Most grantees spoke Afrikaans as their home language ( $86 \%$ or $25 / 29$ ), which was not surprising considering the geographical area in which the study was conducted.

Subjects were asked to describe in their own words why they received a disability grant. The reasons given included: inability to work $(76 \%$ or $22 / 29$ ), the disability grant being their only form of income ( $10 \%$ or $3 / 29)$ or inability to take care of themselves $(17 \%$ or $5 / 29)$. Many grantees explained the nature of their impairment (28\% or $8 / 29$ ). A considerable number of subjects had been disabled since birth (31\% or $9 / 29)$, and $48 \%$. (14/29) had received a permanent disability grant for the past 5 years, while only $7 \%$ $(2 / 29)$ had received a grant for more than 25 years. 
For the remainder of the disability catalogue, subjects were counted as having a disability in a category when they indicated that they had a problem with any activity in that category.

Only three persons (10\% or $3 / 29)$ had just one type of disability (all of them situational disabilities). One person did not fit into any category and the remaining respondents $(86 \%$ or $25 / 29$ ) reported multiple disabilities, i.e. they reported problems with activities in more than one category. It may be cautiously hypothesised that mainly persons with more than one disability qualified for a disability grant in this semi-rural South African area. It is also possible that the budget of the Department of Social Security only permits assistance to those perceived to have the greatest needs, but no such extrapolations are possible on the basis of the current study. Of the grantees, 66\% (19/29) relied on a caregiver to some extent and there was a significant relationship between mean disability score and caregiver utilization $(p=.0001)$. These results will be reported in more detail elsewhere.

A summary of the disability profile is displayed in Table 1 . It was found that most persons had situational disabilities ( $82 \%$ or $24 / 29)$, while only $34 \%$ (10/29) reported personal care disabilities. In spite of $55 \%(16 / 29)$ of the grantees having locomotor disabilities, only a few experienced problems with personal care or dexterity. It is extremely difficult to relate these results to findings from other South African studies. The latter, predominantly prevalence studies, are seldom based on the ICIDH disability catalogue, but rather on varying interpretations and combinations of the concepts of impairment, disability and handicap (www.statssa.gov.za; Schneider et a1, 1999; Coetzee, 1997; Concha and Lorenzo, 1993; Cornieljie et al, 1993; McLaren et al, 1987; Disler et al, 1986).

In the present study, each disability category was analysed individually by looking at the percentage of items or activities reported to be problematic for the grantees, i.e. positive responses. Table 2 illustrates the number of grantees' "yes" responses when asked whether or not they had difficulty with the activity, and includes the percentages

TABLE 1. Number of cases in specific disability categories

\begin{tabular}{|l|c|c|}
\hline \multicolumn{1}{|c|}{ Disability category } & Number of cases & Percentage of cases \\
\hline Situational & 24 & $82 \%$ \\
\hline Communication & 20 & $69 \%$ \\
\hline Body disposition & 18 & $62 \%$ \\
\hline Behavioural & 17 & $59 \%$ \\
\hline Locomotor & 16 & $55 \%$ \\
\hline Dexterity & 11 & $38 \%$ \\
\hline Personal care & 10 & $34 \%$ \\
\hline
\end{tabular}

(rounded off). Only the five most frequently reported items are given in each category, while the remaining items are summed under "other".

\section{Situational disabilities:}

Activities in the situational category refer to activities that are only considered challenging in particular circumstances, such as stressful situations, weather sensitivity or difficulty tolerating light or noise (WHO, 1980). Difficulties with these activities may influence a person's employability, ability to hold down a job or learn a new skill, which in turn may explain the high unemployment rate found in this study (93\% or 27/29).

\section{Communication disabilities:}

Most grantees reported having problems with reading and writing. It was not established whether or not subjects were illiterate. However, as more subjects reported inability to read ( $41 \%$ or $12 / 29)$ and write $(38 \%$ or $11 / 29)$ than inability to see $(31 \%$ or $9 / 29)$, this is a strong possibility, indicating that a variable option "not applicable" would have been useful for the former two activities.

\section{Behavioural disabilities:}

Most of the behavioural disabilities reported, e.g. learning a new skill or knowing what day it is, may explain why the majority of the respondents were also unemployed and reliant on a caregiver, as command of these activities is pivotal for independent living.

\section{Body disposition disabilities:}

Many subjects had difficulties preparing meals, doing laundry and cleaning their homes. This could possibly be related to the fact that the majority of the respondents in this study were men, who are traditionally not involved in household chores. It may also have contributed to the grantees' difficulties in activities in the dexterity, behavioural or situational categories. These relationships were, however not further analysed.

\section{Locomotor disabilities:}

In this category the percentage of grantees reporting difficulties with activities increased as the activities became more complex. Only a few persons reported difficulty moving inside the house $(7 \%$ or $2 / 29)$ or getting out of bed $(7 \%$ or $2 / 29)$, while more persons experienced difficulty with intricate activities such as walking down more than 5 stairs or running.

\section{Dexterity disabilities and personal care disabilities:}

Problems related to personal care and dexterity as indicated by the grantees may all have contributed to their utilisation of caregivers. Although $55 \%$ $(16 / 29)$ of the subjects reported having locomotor disabilities, only a few had problems with activities in the personal care $(34 \%$ or $10 / 29)$ and dexterity categories $(38 \%$ or $11 / 29)$. The ICIDH attributes locomotor disabilities mainly to lower limb impairments, whereas upper limb function is necessary in the latter two categories. Many of the highly prevalent neuro-musculoskeletal impairments, such as stroke, limit activities of both upper and lower limbs, and this discrepancy in the findings was not anticipated by the researcher. 
TABLE 2. Reported difficulties per disability category

\begin{tabular}{|c|c|c|c|}
\hline $\begin{array}{l}\text { Disability } \\
\text { category }\end{array}$ & Item & $\begin{array}{l}\text { Positive } \\
\text { responses }\end{array}$ & $\begin{array}{c}\text { Percentage of } \\
\text { respondents }(n=29)\end{array}$ \\
\hline \multirow{5}{*}{ 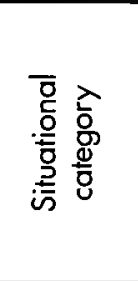 } & Tolerating stress & 20 & $69 \%$ \\
\hline & Tolerating noise & 15 & $52 \%$ \\
\hline & Feeling under the weather & 15 & $52 \%$ \\
\hline & Getting out of breath easily & 13 & $45 \%$ \\
\hline & Tolerating light & 8 & $28 \%$ \\
\hline \multirow{6}{*}{ 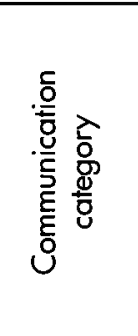 } & Reading & 12 & $41 \%$ \\
\hline & Writing & 11 & $38 \%$ \\
\hline & Seeing & 9 & $31 \%$ \\
\hline & Talking & 8 & $28 \%$ \\
\hline & Understanding & 5 & $17 \%$ \\
\hline & Other (1 item $)$ & 2 & $7 \%$ \\
\hline \multirow{6}{*}{ 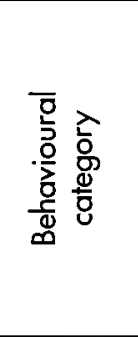 } & Learning a new skill & 14 & $48 \%$ \\
\hline & Knowing what day it is & 12 & $41 \%$ \\
\hline & Making decisions about self & 11 & $38 \%$ \\
\hline & Keeping self safe & 10 & $34 \%$ \\
\hline & Neglecting own appearance & 9 & $31 \%$ \\
\hline & Other (4 items) & 14 & $48 \%$ \\
\hline \multirow{6}{*}{ 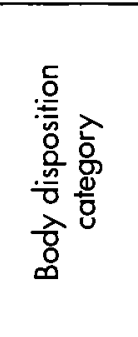 } & Preparing own meal & 12 & $41 \%$ \\
\hline & Cleaning own home & 11 & $38 \%$ \\
\hline & Washing own laundry & 11 & $38 \%$ \\
\hline & Shopping & 10 & $34 \%$ \\
\hline & Picking up objects from the floor & 6 & $21 \%$ \\
\hline & Other (4 items) & 7 & $24 \%$ \\
\hline \multirow{6}{*}{ 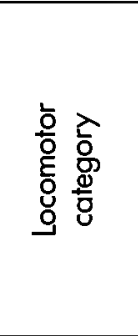 } & Running & 16 & $55 \%$ \\
\hline & Descending $>5$ steps & 9 & $31 \%$ \\
\hline & Ascending $>5$ steps & 9 & $31 \%$ \\
\hline & Descending 2-5 sleps & 5 & $17 \%$ \\
\hline & Ascending $2-5$ steps & 5 & $17 \%$ \\
\hline & Other ( 6 items) & 19 & $66 \%$ \\
\hline \multirow{6}{*}{ 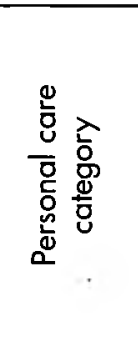 } & Washing self & 9 & $31 \%$ \\
\hline & Dressing self & 6 & $21 \%$ \\
\hline & Shaving self & 4 & $14 \%$ \\
\hline & Controlling stools & 3 & $10 \%$ \\
\hline & Controlling urine & 2 & $7 \%$ \\
\hline & Other (1 item) & 2 & $7 \%$ \\
\hline \multirow{6}{*}{ 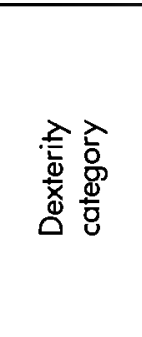 } & Using a telephone & 8 & $28 \%$ \\
\hline & Manipulating small objects & 5 & $17 \%$ \\
\hline & Opening taps & 4 & $14 \%$ \\
\hline & Using taps & 4 & $14 \%$ \\
\hline & Gripping with hands & 3 & $10 \%$ \\
\hline & Other (1 item) & 1 & $3 \%$ \\
\hline
\end{tabular}

\section{CONCLUSIONS:}

The present study succeeded in establishing both demographic data and disability profiles of the study population. In spite of the WHO's intention to provide a classification of disablement via the original ICIDH document, there is little published research utilising the disability categories of this instrument. Many reported figures on disability, most notably the South African census, seem to be impairment based, representing the medical model of disability. This is also true for present legislation regarding eligibility of persons with disabilities for social assistance, as they are primarily assessed on the basis of impairments, rather than disabilities. The Department of Social Security should address this discrepancy and develop more appropriate assessment procedures, reflecting disability and/or handicap within a social model. This is particularly important in view of the world-wide paradigm shift away from a medical model, which presupposes a linear relationship between impairments, disabilities and handicaps.

The disability catalogue of the ICIDH was found to be a relatively easy tool on which to base the questionnaire, although descriptions were found to be cumbersome and categories overlapped somewhat. It remains to be seen whether the revised version (ICIDH-2) will be adopted by the World Health Assembly in May 2001 and lead to more consistency in disability research and, more importantly, whether persons with disabilities will embrace this instrument. It will then be of interest to conduct a study on a similar population using the eight chapter activity catalogue of the ICIDH-2 which replaced the disability catalogue of the original document.

The majority of the respondents in this study $(86 \%$ or $25 / 29)$ reported disabilities in more than one category. It may therefore cautiously be hypothesised that a person only qualifies for a disability grant in South Africa when the disabilities are extensive. This requires further investigation. Furthermore, studies examining aspects relating to the quality of life of persons with multiple disabilities and their caregivers are also recommended, as the present 
quantitative study did not allow for any inferences to be made regarding the impact of multiple disabilities on other aspects of life. For example, the participation catalogue of the new ICIDH-2 could be a useful tool to capture the relevant information about grantees' life-roles.

It was a matter of concern that the present study, although conducted on a relatively small study population, found that nearly one third of the grantees had been disabled since birth. This requires further investigation and also highlights the importance of appropriate primary and secondary prevention programmes as strongly recommended in various South African policy documents (Government Gazette No 1108 of 1997; Mbeki, 1997; Integrated Provincial Disability Strategy, 1999).

Finally, conducting face-to-face interviews in grantees' homes has broadened the researcher's personal and professional perspective in terms of the many challenges facing persons with disabilities who rely on social assistance. She therefore expresses her wholehearted thanks to the grantees and their proxies.

\section{REFERENCES}

Coetzee LT 1997 The prevalence and rehabilitation needs of individuals with locomotor disability in Mitchells Plain. Unpublished thesis $\mathrm{M}$ Phil in Biomedical Engineering, University of Cape Town

Concha ME, Lorenzo T 1993 The prevalence of disability in a rural area of South Africa. South African Journal of Occupational Therapy 23: 6-15

Cornielje H, Ferrinho P, Coetzee D, Reinach SG 1993 Development of a community based rehabilitation programme for a poor urban area in South Africa; Part 1 A disability prevalence study CHASA Journal 4: 26-32

Disler PB, Jacka E, Sayed R, Rip MR, Hurford S, Collis P 1986 The prevalence of locomotor disability and handicap in the Cape Peninsula: parts 1-3. South African Medical Journal 69: 349-357

Government Gazette, Republic of South Africa, Notice 1108 of 1997, White Paper for Social Welfare

ICIDH-2 workshop, 2000 Durban, unpublished proceedings

Integrated Provincial Disability Strategy, Province of the Western Cape: 1999 Draft document, condensed version

Knapp P, Hewison, J 1996 Disagreement in patient and carer assessment of functional disability after stroke. Stroke 30: 934-938
Mbeki T 1997 White Paper on an Integrated National Disability Strategy. Office of the Deputy President

McLaren P, Gear JSS, Irwig LM, Smit AE 1987 Prevalence of motor impairment and disability in a rural community in Kwazulu. International Rehabilitation Medicine 8: 98-104

Sackett DL 1979 Bias in analytical research. Journal of Chronic Diseases 32:51-63

Schneider M, Claasens M, Kimmie R, Naicker S, Roberts A, McLaren P 1999 Community Agency for Social Enquiry. Summary report

Schofield HL, Murphy B, Nankervis J, Singh B, Herman HE, Bloch S 1997 Family carers: Women and men, adult offspring, partners, and parents. Journal of Family Studies 3: 149-169

World Health Organisation 1980 International Classification of Impairments, Disabilities and Handicaps, Geneva

World Health Organisation 1999 International Classification of Functioning and Disability: Beta-2 draft, Full version, Geneva

www.statssa.gov.za

www.who.intllicidh

VRT-3 form: Provincial Administration of the Western Cape. Medical report: Application for/review of social grant for disabled persons and war veterans 